\title{
Notes for genera update - Ascomycota: 6822-6917
}

\author{
Wijayawardene $\mathrm{NN}^{1,2}$, Hyde $\mathrm{KD}^{3}$, McKenzie EHC ${ }^{4}$, Wang $\mathrm{Y}^{1 *}$
}

${ }^{1}$ Department of Plant Pathology, Agriculture College, Guizhou University, Guiyang 550025, People's Republic of China ${ }^{2}$ Guizhou Key Laboratory Agro-Bioengineering, Guizhou University, Guiyang, Guizhou 550025, China ${ }^{3}$ Center of Excellence in Fungal Research, Mae Fah Luang University, Chiang Rai, 57100, Thailand

${ }^{4}$ Manaaki Whenua Landcare Research, Private Bag 92170, Auckland, New Zealand

Wijayawardene NN, Hyde KD, McKenzie EHC, Wang Y 2018 - Notes for genera update Ascomycota: 6822-6917. Mycosphere 9(6), 1222-1234, Doi 10.5943/mycosphere/9/6/11

\begin{abstract}
Acquiring updated information of fungi is one of the challenges faced by mycologists. This study is a continuation of Wijayawardene et al. $(2017,2018)$ and provides notes (generic name, classification, number of species, typification details, life mode, distribution, references) for each genus of Ascomycota described mainly during the period January to June 2018.
\end{abstract}

Key words - Classification - Coelomycetes - Hyphomycetes - New Genera - Sexual Genera

\section{Introduction}

Proposing one name for pleomorphic genera, reinstating synonymized genera, and synonymizing genera based on phylogenetic data are common practice among taxonomists. Moreover, re-visiting old genera by observing type materials and illustrating them is also progressing (e.g. Boonmee et al. 2017). Hence, the importance of compiling all taxonomic (including taxonomic ranks) and nomenclature data since Kirk et al. (2008) was emphasized in Wijayawardene et al. (2017), and has been continuously updated as a series of notes (Wijayawardene et al. 2018).

\section{Materials \& Methods}

We follow the format used by Wijayawardene et al. $(2017,2018)$ to prepare the entries for each genus. The list of recently introduced genera (January 2018-June 2018) has been extracted from Index Fungorum (2018).

\section{Notes for genera}

Acericola Wanas., Camporesi, E.B.G. Jones \& K.D. Hyde 2017, Phaeosphaeriaceae, Pleosporales, Dothideomycetes, one species, type: A. italica Wanas., Camporesi, E.B.G. Jones \& K.D. Hyde, asexual morph unknown, saprobes, Italy, see Hyde et al. (2017; taxonomy), Wijayawardene et al. (2018; outline), cultures and sequences are available.

Aculeata W. Dong, H. Zhang \& K.D. Hyde 2018, Herpotrichiellaceae, Chaetothyriales, Eurotiomycetes, one species, type: A. aquatica W. Dong, H. Zhang \& K.D. Hyde, asexual morph unknown, saprobes, aquatic, Thailand, see Dong et al. (2018; taxonomy), cultures and sequences are available. 
Allocryptovalsa Senwanna, Phookamsak \& K.D. Hyde 2017, Valsaceae, Diaporthales, Sordariomycetes, three species, type: A. polyspora Senwanna, Phookamsak \& K.D. Hyde, asexual morph unknown, saprobes, Thailand, see Senwanna et al. (2017; taxonomy), cultures and sequences are available.

Apiognomonioides U. Braun, J.Z. Groenew. \& Crous 2018, Tubakiaceae, Diaporthales, Sordariomycetes, one species, type: A. supraseptata (Kaneko \& Kobayashi) U. Braun, J.Z. Groenew. $\&$ Crous, asexual morph unknown, saprobes, Japan, see Braun et al. (2018; taxonomy), cultures and sequences are available.

Aquacidia Aptroot 2018, Pilocarpaceae, Lecanorales, Lecanoromycetes, three species, type: A. trachona (Ach.) Aptroot, asexual morph unknown, lichenized, cosmopolitan, see Aptroot et al. (2018; taxonomy), cultures and sequences are available.

Aquadictyospora Z.L. Luo, K.D. Hyde \& H.Y. Su 2017, Dictyosporiaceae, Pleosporales, Dothideomycetes, one species, type: A. lignicola Z.L. Luo, W.L. Li, K.D. Hyde \& H.Y. Su, hyphomycetous, sexual morph unknown, saprobes, aquatic, China, see Li et al. (2017; taxonomy), cultures and sequences are available.

Araucasphaeria Crous \& M.J. Wingf. 2018, Teratosphaeriaceae, Capnodiales, Dothideomycetes, one species, type: A. foliorum Crous \& M.J. Wingf., asexual morph unknown, saprobes, terrestrial, Chile, see Crous et al. (2018; taxonomy), cultures and sequences are available.

Arxotrichum A. Nováková \& M. Kolařik 2018, Chaetomiaceae, Sordariales, Sordariomycetes, two species, type: A. wyomingense A. Nováková \& M. Kolařik, hyphomycetous, saprobes, terrestrial, USA, see Crous et al. (2018; taxonomy), cultures and sequences are available.

Bhatiellae Wanas., Camporesi \& K.D. Hyde 2018, Phaeosphaeriaceae, Pleosporales, Dothideomycetes, one species, type: B. rosae Wanas., Camporesi, E.B.G. Jones \& K.D. Hyde, hyphomycetous, sexual morph unknown, saprobes, Italy, see Wanasinghe et al. (2018; taxonomy), cultures and sequences are available.

Brevicollum Kaz. Tanaka, A. Hashim. \& Toy. Sato 2017, Neohendersoniaceae, Pleosporales, Dothideomycetes, two species, type: B. hyalosporum Kaz. Tanaka \& Toy. Sato, asexual morph unknown, bryophilous, cosmopolitan, see Tanaka et al. (2017; taxonomy), cultures and sequences are available.

Caeruleoconidia Zhurb. \& Pino-Bodas 2017, Ascomycota genera incertae sedis, two species, type: C. ochrolechiae Zhurb. \& Pino-Bodas, coelomycetous, sexual morph unknown, lichenicolous, Asia, see Zhurbenko et al. (2015; taxonomy, validation), Zhurbenko \& Pino-Bodas (2017; lichenicolous taxa on Cladonia), Wijayawardene et al. (2016, 2018; taxonomy, outline), cultures and sequences are available.

Camarosporomyces Crous 2017, Camarosporiaceae, Pleosporales, Dothideomycetes, one species, type: C. flavigenus (Constant. \& Aa) Crous, coelomycetous, sexual morph unknown, saprobes, Romania, see Crous \& Groenewald (2017; taxonomy, phylogeny), Wanasinghe et al. (2017; taxonomy), Wijayawardene et al. (2017, 2018; outline), cultures and sequences are available.

Chiangraiomyces Senan. \& K.D. Hyde 2017, Diaporthaceae, Diaporthales, Sordariomycetes, one species, type: C. bauhiniae Senan. \& K.D. Hyde, asexual morph coelomycetous, saprobes, Thailand, see Senanayake et al. (2017, 2018; taxonomy), Wijayawardene et al. (2018; outline), cultures and sequences are available. 
Cochlearomyces Crous 2017, Cochlearomycetaceae, Helotiales, Leotiomycetes, one species, type: C. eucalypti Crous, coelomycetous, sexual morph unknown, saprobes, Australia, see Crous et al. (2017; taxonomy), cultures and sequences are available.

Corticimorbus S.F. Chen \& M.J. Wingf. 2016, Cryphonectriaceae, Diaporthales, Sordariomycetes, one species, type: $C$. sinomyrti S.F. Chen, F.F. Liu \& M.J. Wingf., asexual morph coelomycetous, pathogens, China, see Chen et al. (2016; taxonomy, nomenclature), cultures and sequences are available.

Cycasicola Wanas., E.B.G. Jones \& K.D. Hyde 2018, Thyridariaceae, Pleosporales, Dothideomycetes, two species, type: C. goaensis Wanas., E.B.G. Jones \& K.D. Hyde, coelomycetous, sexual morph unknown, saprobes, India, see Wanasinghe et al. (2018; taxonomy), cultures and sequences are available.

Dactylidina Wanas., Camporesi \& K.D. Hyde 2018, Phaeosphaeriaceae, Pleosporales, Dothideomycetes, two species, type: D. dactylidis (Wanas., Camporesi, E.B.G. Jones \& K.D. Hyde) Wanas. \& K.D. Hyde, asexual morph unknown, saprobes, Italy, see Wanasinghe et al. (2018; taxonomy), cultures and sequences are available.

Dictyosporina L.M. Abreu, R.F. Castañeda \& O.L. Pereira 2017, Mycosphaerellaceae, Mycosphaerellales, Dothideomycetes, one species, type: D. ferruginea L.M. Abreu, R.F. Castañeda \& O.L. Pereira, hyphomycetous, sexual morph unknown, saprobes, Brazil, see Hyde et al. (2017; taxonomy, phylogeny), Wijayawardene et al. (2017, 2018; outline), ex-type living culture COAD 2272.

Didysimulans Tibpromma, Camporesi \& K.D. Hyde 2017, Didymellaceae, Pleosporales, Dothideomycetes, two species, type: D. mezzanensis Tibpromma, Camporesi \& K.D. Hyde, asexual morph hyphomycetous, saprobes, Italy, see Tibpromma et al. (2017; taxonomy, phylogeny), Wijayawardene et al. (2017, 2018; outline), cultures and sequences are available.

Dlhawksworthia Wanas., Camporesi \& K.D. Hyde 2018 (as Hawksworthiana Wanas., Camporesi \& K.D. Hyde), Phaeosphaeriaceae, Pleosporales, Dothideomycetes, three species, type: D. alliariae (Thambug., Camporesi \& K.D. Hyde) Wanas. \& K.D. Hyde, asexual morph coelomycetous, saprobes, Italy, see Wanasinghe et al. (2018; taxonomy, nomenclature), Wanasinghe \& Hyde (2018), cultures and sequences are available.

Ducatina Ertz \& Søchting 2017, Trapeliaceae, Baeomycetales, Lecanoromycetes, one species, type: D. umbilicata Ertz \& Søchting, asexual morph unknown, lichenized, Crozet Islands, see Ertz et al. (2017b; taxonomy), cultures and sequences are available.

Embarria Wanas., Camporesi \& K.D. Hyde 2018, Phaeosphaeriaceae, Pleosporales, Dothideomycetes, one species, type: E. clematidis (Wanas., Camporesi, E.B.G. Jones \& K.D. Hyde) Wanas. \& K.D. Hyde, asexual morph unknown, saprobes, Italy, see Wanasinghe et al. (2018; taxonomy), cultures and sequences are available.

Fitzroyomyces Crous 2017, Stictidaceae, Ostropales, Lecanoromycetes, one species, type: F. cyperi Crous, coelomycetous, sexual morph unknown, saprobes, Australia, see Crous et al. (2017; taxonomy), cultures and sequences are available.

Glutinomyces Nor. Nakam. 2017, Hyaloscyphaceae, Helotiales, Leotiomycetes, one species, type: G. brunneus Nor. Nakam., hyphomycetous, sexual morph unknown, saprobes, Japan, see Crous et al. (2017; taxonomy), cultures and sequences are available. 
Halobyssothecium Dayarathne, E.B.G. Jones \& K.D. Hyde 2018, Lentitheciaceae, Pleosporales, Dothideomycetes, one species, type: H. obiones (M.E. Barr) Dayarathne, E.B.G. Jones \& K.D. Hyde, asexual morph unknown, saprobes, terrestrial, UK, see Dayarathne et al. (2018; taxonomy), cultures and sequences are available.

Helicotubeufia Y.Z. Lu \& J.K. Liu 2018, Tubeufiaceae, Tubeufiales, Dothideomycetes, three species, type: H. guangxiensis Y.Z. Lu \& J.K. Liu, asexual morph hyphomycetous, saprobes, aquatic, China, see Liu et al. (2018; taxonomy), cultures and sequences are available.

Henssenia Ertz, R.S. Poulsen \& Søchting 2017, Koerberiaceae, Peltigerales, Lecanoromycetes, four species, type: H. glaucella (Tuck.) Ertz, R.S. Poulsen \& Søchting, asexual morph unknown, lichenized, cosmopolitan, see Ertz et al. (2017a; taxonomy), cultures and sequences are available.

Hyaliappendispora Senan., Camporesi \& K.D. Hyde 2017, Diaporthaceae, Diaporthales, Sordariomycetes, one species, type: H. galii Senan., Camporesi \& K.D. Hyde, asexual morph coelomycetous, saprobes, Italy, see Senanayake et al. (2017, 2018; taxonomy), Wijayawardene et al. (2018; outline), cultures and sequences are available.

Idriellomyces Crous 2018, Phlogicylindriaceae, Xylariales, Sordariomycetes, two species, type: I. eucalypti Crous, hyphomycetous, saprobes, terrestrial, Australia, see Crous et al. (2018; taxonomy), cultures and sequences are available.

Involutiscutellula U. Braun \& C. Nakash. 2018, Tubakiaceae, Diaporthales, Sordariomycetes, one species, type: I. rubra (T. Yokoy. \& Tubaki) U. Braun \& C. Nakash., coelomycetous, sexual morph unknown, endophytes, or rarely as weak pathogens, saprobes, Asia, see Braun et al. (2018; taxonomy), cultures and sequences are available.

Italica Wanas., Camporesi \& K.D. Hyde 2018, Phaeosphaeriaceae, Pleosporales, Dothideomycetes, one species, type: I. luzulae (Wanas., Camporesi, E.B.G. Jones \& K.D. Hyde) Wanas. \& K.D. Hyde, asexual morph unknown, saprobes, Italy, see Wanasinghe et al. (2018; taxonomy), cultures and sequences are available.

Linearistroma Höhn. 1910, Clavicipitaceae, Hypocreales, Sordariomycetes, one species, type: $C$. lineare (Rehm) Höhn., asexual morph unknown, saprobes, Asia, South America, see Jaklitsch et al. (2016; outline), cultures and sequences are unavailable.

Longitudinalis Tibpromma \& K.D. Hyde 2017, Plectosphaerellaceae, Glomerellales, Sordariomycetes, one species, type: L. nabanheensis Tibpromma \& K.D. Hyde, hyphomycetous, sexual morph unknown, saprobes, China, see Hyde et al. (2017; taxonomy), Wijayawardene et al. (2018; outline), cultures and sequences are available.

Marjia Wanas., Gafforov \& K.D. Hyde 2018, Melanommataceae, Pleosporales, Dothideomycetes, two species, type: $M$. tianschanica Wanas., Gafforov \& K.D. Hyde, asexual morph unknown, saprobes, Uzbekistan, see Wanasinghe et al. (2018; taxonomy), cultures and sequences are available.

Marquesius L.B. Conç. et al. 2018, Dothideomycetes genera incertae sedis, one species, type: $M$. aquaticus L.B. Conc., R.F. Castañeda \& Gusmao, hyphomycetous, saprobes, aquatic, Brazil, see Crous et al. (2018; taxonomy), cultures and sequences are available.

Marsupiomyces Senan. \& K.D. Hyde 2017, Diaporthaceae, Diaporthales, Sordariomycetes, two species, type: M. epidermoideus Perera, Senan., Bulgakov \& K.D. Hyde, asexual morph unknown, 
saprobes, Italy, Russia, see Senanayake et al. (2017, 2018; taxonomy), Wijayawardene et al. (2018; outline), cultures and sequences are available.

Megatomentella D.A.C. Almeida, Gusmão \& A.N. Mill. 2017, Pleosporales genera incertae sedis, Dothideomycetes, one species, type: M. cristata D.A.C. Almeida, Gusmão \& A.N. Mill., asexual morph unknown, saprobes, Brazil, see Crous et al. (2017; taxonomy), cultures and sequences are available.

Melanocucurbitaria Wanas., Gafforov \& K.D. Hyde 2018, Melanommataceae, Pleosporales, Dothideomycetes, one species, type: M. uzbekistanica Wanas., Gafforov \& K.D. Hyde, asexual morph unknown, saprobes, Uzbekistan, see Wanasinghe et al. (2018; taxonomy), cultures and sequences are available.

Melanodiplodia Wanas., Gafforov \& K.D. Hyde 2018, Melanommataceae, Pleosporales, Dothideomycetes, one species, type: $M$. tianschanica Wanas., Gafforov \& K.D. Hyde, coelomycetous, sexual morph unknown, saprobes, Uzbekistan, see Wanasinghe et al. (2018; taxonomy), cultures and sequences are available.

Microascospora Senan. \& K.D. Hyde 2017, Diaporthaceae, Diaporthales, Sordariomycetes, one species, type: $M$. rubi Senan., Camporesi \& K.D. Hyde, asexual morph unknown, saprobes, Italy, see Senanayake et al. (2017, 2018; taxonomy), Wijayawardene et al. (2018; outline), cultures and sequences are available.

Monoseptella Wanas., Gafforov \& K.D. Hyde 2018, Melanommataceae, Pleosporales, Dothideomycetes, one species, type: M. rosae Wanas., Gafforov \& K.D. Hyde, asexual morph unknown, saprobes, Uzbekistan, see Wanasinghe et al. (2018; taxonomy), cultures and sequences are available.

Murramarangomyces Crous 2017, Murramarangomycetaceae, Murramarangomycetales, Dothideomycetes, one species, type: $M$. corymbiae Crous, sexual morph unknown, saprobes, Australia, see Crous et al. (2017; taxonomy), cultures and sequences are available.

Myrotheciomyces Crous 2018, Myrotheciomycetaceae, Hypocreales, Sordariomycetes, one species, type: M. corymbiae Crous, hyphomycetous, saprobes, terrestrial, Australia, see Crous et al. (2018; taxonomy), cultures and sequences are available.

Neoanungitea Crous 2017, Microthyriaceae, Microthyriales, Dothideomycetes, one species, type: N. eucalypti Crous, hyphomycetous, sexual morph unknown, saprobes, Australia, see Crous et al. (2017; taxonomy), cultures and sequences are available.

Neoconiothyrium Crous 2017, Coniothyriaceae, Pleosporales, Dothideomycetes, four species, type: $N$. persooniae Crous, coelomycetes, sexual morph unknown, saprobes, Australia, see Crous et al. (2017; taxonomy), Wanasinghe et al. (2018; new species), cultures and sequences are available.

Neocucurbitaria Wanas., E.B.G. Jones \& K.D. Hyde 2017, Cucurbitariaceae, Pleosporales, Dothideomycetes, 19 species, type: N. unguis-hominis (Punith. \& M.P. English) Wanas., E.B.G. Jones \& K.D. Hyde, asexual morph coelomycetous, saprobes, cosmopolitan, see Jaklitsch et al. (2017; new species, phylogeny), Valenzuela-Lopez et al. (2017; new species, phylogeny), Wanasinghe et al. (2017; taxonomy, phylogeny), Wijayawardene et al. (2017, 2018; outline), cultures and sequences are available. 
Neohelicosporium Y.Z. Lu, J.C. Kang \& K.D. Hyde 2018, Tubeufiaceae, Tubeufiales, Dothideomycetes, six species, type: N. parvisporum Y.Z. Lu, J.C. Kang \& K.D. Hyde, hyphomycetous, sexual morph unknown, saprobes, aquatic, China, see Lu et al. (2018; taxonomy), cultures and sequences are available.

Neolauriomyces Crous 2018, Neolauriomycetaceae, Helotiales, Leotiomycetes, one species, type: $N$. eucalypti Crous, hyphomycetous, saprobes, terrestrial, Australia, see Crous et al. (2018; taxonomy), cultures and sequences are available.

Neometulocladosporiella Crous \& M.J. Wingf. 2018, Rutstroemiaceae, Helotiales, Leotiomycetes, one species, type: N. eucalypti Crous \& M.J. Wingf., hyphomycetous, saprobes, terrestrial, Colombia, see Crous et al. (2018; taxonomy), cultures and sequences are available.

Neopaucispora Wanas., Gafforov \& K.D. Hyde 2018, Lophiostomataceae, Pleosporales, Dothideomycetes, one species, type: N. rosaecae Wanas., Gafforov \& K.D. Hyde, asexual morph unknown, saprobes, Uzbekistan, see Wanasinghe et al. (2018; taxonomy), cultures and sequences are available.

Neophyllachora Dayar. \& K.D. Hyde 2017, Phyllachoraceae, Phyllachorales, Sordariomycetes, four species, type: N. myrciae (Lév.) Dayar. \& K.D. Hyde, asexual morph coelomycetous, saprobes, cosmopolitan, see Dayarathne et al. (2017; taxonomy), cultures and sequences are available.

Neopyrenochaeta Valenz.-Lopez, Crous, Stchigel, Guarro \& J.F. Cano 2017, Neopyrenochaetaceae, Pleosporales, Dothideomycetes, four species, type: N. acicola (Moug. \& Lév.) Valenz.-Lopez, Crous, Stchigel, Guarro \& J.F. Cano, coelomycetous, saprobes, terrestrial or aquatic, The Netherlands, South Africa, India, see Valenzuela-Lopez et al. (2017; taxonomy), cultures and sequences are available.

Neopyrenochaetopsis Valenz-Lopez, J.F. Cano, Guarro \& Stchigel 2017, Pyrenochaetopsidaceae, Pleosporales, Dothideomycetes, one species, type: P. hominis Valenz.-Lopez, J.F. Cano, Guarro \& Stchigel, coelomycetous, from human skin, terrestrial, USA, see Valenzuela-Lopez et al. (2017; taxonomy), cultures and sequences are available.

Neoramichloridium Phook., Thambug. \& K.D. Hyde 2017, Mycosphaerellaceae, Mycosphaerellales, Dothideomycetes, one species, type: N. bambusicola Thambug., Phook. \& K.D. Hyde, hyphomycetous, sexual morph unknown, saprobes, Thailand, see Thambugala et al. (2017; taxonomy, phylogeny), Wijayawardene et al. (2017, 2018; outline), cultures and sequences are available.

Nullicamyces Crous 2018, Chaetothyriaceae, Chaetothyriales, Eurotiomycetes, one species, type: $N$. eucalypti Crous, hyphomycetous, saprobes, terrestrial, Australia, see Crous et al. (2018; taxonomy), cultures and sequences are available.

Oblongisporothyrium U. Braun \& C. Naksh. 2018, Tubakiaceae, Diaporthales, Sordariomycetes, one species, type: O. castanopsidis (T. Yokoy. \& Tubaki) U. Braun \& C. Nakash., coelomycetous, sexual morph unknown, endophytes or saprobes, Asia (Japan), see Braun et al. (2018; taxonomy), cultures and sequences are available.

Paracladophialophora Crous 2016, Paracladophialophoraceae, Chaetothyriales, Eurotiomycetes, two species, type: P. carceris Crous \& Roets, hyphomycetous, saprobes, terrestrial, Australia, see Crous et al. (2016; taxonomy), cultures and sequences are available. 
Paradiaporthe Senan., Camporesi \& K.D. Hyde 2017, Diaporthaceae, Diaporthales, Sordariomycetes, one species, type: P. artemisiae Senan., Camporesi \& K.D. Hyde, asexual morph unknown, saprobes, Italy, see Senanayake et al. (2017, 2018; taxonomy), Wijayawardene et al. (2018; outline), cultures and sequences are available.

Paraopeba V.P. Abreu, A.A.M. Gomes, Firmino \& O.L. Pereira 2017, Asterinaceae, Asterinales, Dothideomycetes, one species, type: P. schefflerae V.P. Abreu, A.A.M. Gomes, Firmino \& O.L. Pereira, hyphomycetous, sexual morph unknown, saprobes, Brazil, see Crous et al. (2017; taxonomy), cultures and sequences are available.

Paraphysalospora Crous 2017, Clypeophysalosporaceae, Xylariales, Sordariomycetes, three species, type: P. eucalypti Crous, asexual morph hyphomycetous, saprobes, Australia, see Crous et al. (2017; taxonomy), cultures and sequences are available.

Parapyrenochaeta Valenz.-Lopez, Crous, Stchigel, Guarro \& J.F. Cano 2017, Parapyrenochaetaceae, Pleosporales, Dothideomycetes, two species, type: P. protearum (Crous) Valenz.-Lopez, Crous, Stchigel, Guarro \& J.F. Cano, coelomycetous, saprobes or pathogens, terrestrial, Australia, South Africa, France, see Valenzuela-Lopez et al. (2017; taxonomy), cultures and sequences are available.

Pararamichloridium Crous 2017, Pararamichloridiaceae, Pararamichloridiales, Sordariomycetes, two species, type: $P$. livistonae Crous, hyphomycetous, sexual morph unknown, saprobes, Australia, India, see Crous et al. (2017; taxonomy), cultures and sequences are available.

Pararoussoella Wanas., E.B.G. Jones \& K.D. Hyde 2018, Thyridariaceae, Pleosporales, Dothideomycetes, one species, type: P. rosarum Wanas., E.B.G. Jones \& K.D. Hyde, asexual morph unknown, saprobes, UK, see Wanasinghe et al. (2018; taxonomy, nomenclature), cultures and sequences are available.

Parastagonosporella M. Bakhshi, Arzanlou \& Crous 2018, Phaeosphaeriaceae, Pleosporales, Dothideomycetes, one species, type: P. allopiae M. Bakhshi, Arzanlou \& Crous, coelomycetous, sexual morph unknown, saprobes, terrestrial, UK, see Bakhshi et al. (2018; taxonomy), cultures and sequences are available.

Paratubakia 2018 U. Braun \& C. Nakash., Tubakiaceae, Diaporthales, Sordariomycetes, two species, type: P. subglobosa (T. Yokoy. \& Tubaki) U. Braun \& C. Nakash., coelomycetous, sexual morph unknown, endophytes, Asia (Japan), see Braun et al. (2018; taxonomy), cultures and sequences are available.

Paraxylaria Wanas., Gafforov, E.B.G. Jones \& K.D. Hyde 2018, Coniocessiaceae, Xylariales, Sordariomycetes, one species, type: P. rosacearum Wanas., Gafforov, E.B.G. Jones \& K.D. Hyde, asexual morph unknown, saprobes, Uzbekistan, see Wanasinghe et al. (2018; taxonomy, nomenclature), cultures and sequences are available.

Phaeoappendicospora Senan., Q.R. Li \& K.D. Hyde 2017, Diaporthales genera incertae sedis, Sordariomycetes, one species, type: P. thailandensis Q.R. Li, Senan. \& K.D. Hyde, asexual morph unknown, saprobes, Thailand, see Senanayake et al. (2017, 2018; taxonomy), Wijayawardene et al. (2018; outline), cultures and sequences are available.

Pileospora J.B. Tanney \& K.A. Seifert 2018, Phaeosphaeriaceae, Pleosporales, Dothideomycetes, one species, type: P. piceae J.B. Tanney \& K.A. Seifert, coelomycetous, sexual morph unknown, 
saprobes, terrestrial, Canada, see Tanney \& Seifert (2018; taxonomy), cultures and sequences are available.

Planamyces Crous \& Decock 2017, Pyronemataceae, Pezizales, Pezizomycetes, one species, $P$. parisiensis Crous \& Decock, hyphomycetous, sexual morph unknown, saprobes, France, see Crous et al. (2017; taxonomy), cultures and sequences are available.

Pleiocarpon L. Lombard \& D. Aiello 2017, Nectriaceae, Hypocreales, Sordariomycetes, one species, type: P. strelitziae L. Lombard \& D. Aiello, hyphomycetous, sexual morph unknown, pathogens, Italy, see Aiello et al. (2017; taxonomy), Wijayawardene et al. (2018; outline), cultures and sequences are available.

Porodiplodia Crous 2018, Porodiplodiaceae, Helotiales, Leotiomycetes, one species, type: P. livistonae Crous, hyphomycetous, saprobes, terrestrial, Australia, see Crous et al. (2018; taxonomy), cultures and sequences are available.

Pseudoanungitea Crous 2018, Venturiaceae, Venturiales, Dothideomycetes, three species, type: $P$. syzygii (Crous et al.) Crous, hyphomycetes, sexual morph unknown, saprobes, South Africa, Europe, see Braun et al. (2018; taxonomy), cultures and sequences are available.

Pseudoarthrographis Crous \& Thangavel 2018, Dothideomycetes genera incertae sedis, one species, type: P. phlogis Crous \& Thangavel, hyphomycetous, saprobes, terrestrial, New Zealand, see Crous et al. (2018; taxonomy), cultures and sequences are available.

Pseudobyssosphaeria H.B. Jiang \& K.D. Hyde 2018, Melanommataceae, Pleosporales, Dothideomycetes, one species, type: P. bambusae H.B. Jiang \& K.D. Hyde, asexual morph unknown, saprobes, Thailand, see Hyde et al. (2018; taxonomy), cultures and sequences are available.

Pseudodactylaria Crous 2017, Pararamichloridiaceae, Pararamichloridiales, Sordariomycetes, two species, $P$. xanthorrhoeae Crous, hyphomycetous, sexual morph unknown, saprobes, Australia, Hong Kong, see Crous et al. (2017; taxonomy), cultures and sequences are available.

Pseudoneoconiothyrium Wanas., Phukhams., Camporesi \& K.D. Hyde 2018 (as Neoconiothyrium Wanas., Phukhams., Camporesi \& K.D. Hyde fide Wanasinghe et al. 2018), Thyridariaceae, Pleosporales, Dothideomycetes, one species, type: P. rosae (Phukhams., Camporesi \& K.D. Hyde) Phukhams., Camporesi \& K.D. Hyde, coelomycetous, sexual morph unknown, saprobes, Italy, see Wanasinghe et al. (2018; taxonomy), Wanasinghe \& Hyde (2018; nomenclature), cultures and sequences are available.

Pseudopyrenochaeta Valenz.-Lopez, Crous, Stchigel, Guarro \& J.F. Cano 2017, Pseudopyrenochaetaceae, Pleosporales, Dothideomycetes, two species, type: P. lycopersici (R.W. Schneid. \& Gerlach) Valenz.-Lopez, Crous, Stchigel, Guarro \& J.F. Cano, coelomycetous, associated with soil and roots, terrestrial, Germany, The Netherlands, see Valenzuela-Lopez et al. (2017; taxonomy), cultures and sequences are available.

Pseudostanjehughesia J. Yang \& K.D. Hyde 2017, Diaporthales genera incertae sedis, Sordariomycetes, one species, type: P. aquitropica J. Yang \& K.D. Hyde, hyphomycetous, sexual morph unknown, aquatic, saprobes, Thailand, see Yang et al. (2017; taxonomy), Wijayawardene et al. (2018; outline), cultures and sequences are available.

Pseudosubramaniomyces Crous 2017, Xylariales genera incertae sedis, Sordariomycetes, one species, type: P. fusisaprophyticus (Matsush.) Crous., hyphomycetous, sexual morph unknown, from 
soil, Australia, Papua New Guinea, see Crous et al. (2017; taxonomy), cultures and sequences are available.

Psychronectria J. Pawłowska, Istel, Wrzosek, D. Hawksw. 2017, Tilachlidiaceae, Hypocreales, Sordariomycetes, one species, type: P. hyperantarctica (D. Hawksw.) J. Pawłowska, Istel, Wrzosek \& D. Hawksw., asexual morph unknown, bryophilous, cosmopolitan, see Pawłowska et al. (2017; taxonomy), cultures and sequences are available.

Purpurepithecium Jayasiri \& K.D. Hyde 2017, Gloniaceae, Mytilinidiales, Dothideomycetes, one species, type: P. murisporum Jayasiri \& K.D. Hyde, asexual morph hyphomycetous, saprobes, Italy, see Jayasiri et al. (2017; taxonomy, phylogeny), Wijayawardene et al. (2017, 2018; outline), cultures and sequences are available.

Querciphoma Crous 2017, Leptosphaeriaceae, Pleosporales, Dothideomycetes, two species, type: Q. minuta (J.C. Carter) Crous \& P.M. Kirk, coelomycetous, sexual morph unknown, saprobes, USA, see Crous \& Groenewald (2017; taxonomy), Wijayawardene et al. (2017, 2018; outline), cultures and sequences are available.

Quixadomyces Cantillo \& Gusmão 2018, Parapyrenochaetaceae, Pleosporales, Dothideomycetes, one species, type: $Q$. cearensis Cantillo \& Gusmão, coelomycetous, saprobes, terrestrial, Brazil, see Crous et al. (2018; taxonomy), cultures and sequences are available.

Racheliella Crous \& U. Braun 2018, Tubakiaceae, Diaporthales, Sordariomycetes, two species, type: $R$. wingfieldiana Crous \& U. Braun, coesexual morph unknown, saprobes and plant pathogens, South Africa, Thailand, see Braun et al. (2018; taxonomy), cultures and sequences are available.

Sammeyersia S.Y. Guo, E.B.G. Jones \& K.L. Pang 2017, Lulworthiaceae, Lulworthiales, Sordariomycetes, one species, type: S. grandispora (Meyers) S.Y. Guo, E.B.G. Jones \& K.L. Pang, asexual morph unknown, saprobes, cosmopolitan, see Abdel-Wahab et al. (2017; taxonomy), cultures and sequences are available.

Saprothyrium U. Braun, Crous \& J.Z. Groenew. 2018, Tubakiaceae, Diaporthales, Sordariomycetes, one species, type: S. thailandense (Senan. et al.) U. Braun, Crous \& J.Z. Groenew., coelomycetous, sexual morph unknown, saprobes, Thailand, see Braun et al. (2018; taxonomy), cultures and sequences are available.

Snippocia Ertz, Kukwa \& Sanderson 2018, Arthoniaceae, Arthoniales, Arthoniomycetes, one species, type: S. nivea (D. Hawksw. \& P. James) Ertz \& Sanderson, asexual morph unknown, lichenized, Europe, see Ertz et al. (2018; taxonomy), cultures and sequences are available.

Sphaerosporithyrium U. Braun, Crous, O. Moreno-Rico \& Marm. 2018, Tubakiaceae, Diaporthales, Sordariomycetes, one species, type: S. mexicanum O. Moreno-Rico, U. Braun \& Marm., coelomycetes, sexual morph unknown, saprobes, Mexico, see Braun et al. (2018; taxonomy), cultures and sequences are available.

Sporormurispora Wanas., Bulgakov, Gafforov \& K.D. Hyde 2018, Sporormiaceae, Pleosporales, Dothideomycetes, two species, type: S. atraphaxidis Wanas., Bulgakov, E.B.G. Jones \& K.D. Hyde, asexual morph unknown, saprobes, Russia, Uzbekistan, see Wanasinghe et al. (2018; taxonomy), cultures and sequences are available.

Translucidithyrium X.Y. Zeng \& K.D. Hyde 2018, Phaeothecoidiellaceae, Capnodiales, Dothideomycetes, one species, type: T. thailandicum X.Y. Zeng \& K.D. Hyde, asexual morph 
unknown, on leaves (sooty moulds), terrestrial, Thailand, see Zeng et al. (2018; taxonomy), cultures and sequences are available.

Uzbekistanica Wanas., Gafforov \& K.D. Hyde 2018, Melanommataceae, Pleosporales, Dothideomycetes, two species, type: U. rosae-hissaricae Wanas., Gafforov \& K.D. Hyde, asexual morph coelomycetous, saprobes, Uzbekistan, see Wanasinghe et al. (2018; taxonomy), cultures and sequences are available.

Vandijckella Sandoval-Denis 2017, Vandijckellaceae, Helotiales, Leotiomycetes, one species, type: $V$. johannae Sandoval-Denis., hyphomycetous, sexual morph unknown, from soil, The Netherlands, see Crous et al. (2017; taxonomy), cultures and sequences are available.

Verhulstia Hern.-Rest. 2017, Chaetosphaeriaceae, Chaetosphaeriales, Sordariomycetes, one species, type: V. trisororum Hern.-Rest., hyphomycetous, sexual morph unknown, from soil, The Netherlands, see Crous et al. (2017; taxonomy), cultures and sequences are available.

Xenomassariosphaeria Jayasiri, Wanas. \& K.D. Hyde 2018, Paradictyoarthriniaceae, Pleosporales, Dothideomycetes, one species, type: X. rosae Jayasiri, Camporesi, \& K.D. Hyde, asexual morph unknown, saprobes, Italy, see Wanasinghe et al. (2018; taxonomy), cultures and sequences are available.

Xenopassalora Crous 2017, Mycosphaerellaceae, Capnodiales, Dothideomycetes, two species, type: $X$. petrophiles Crous, hyphomycetous, sexual morph unknown, phytopathogens, Australia, see Crous et al. (2017; taxonomy), cultures and sequences are available.

Yunnanensis Karun., Phook. \& K.D. Hyde 2017, Phaeosphaeriaceae, Pleosporales, Dothideomycetes, three species, type: Y. phragmitis Karun., Phook. \& K.D. Hyde, asexual morph coelomycetous, saprobes, China, see Karunarathna et al. (2017; taxonomy), cultures and sequences are available.

\section{Acknowledgements}

Nalin Wijayawardene thanks National Institute of Fundamental Studies, Kandy, Sri Lanka for the position of visiting scientist during the tenure of which this paper was finalised. The research is partially supported by Mushroom Research Foundation, Thailand, the projects of National Natural Science Foundation of China (No. 31500451, 31560536 and 31560489), Guizhou science and technology department international cooperation base project ([2018]5806), Guizhou Province Education Department Youth Science and technology talent growth project (2016-120) and Bijie Science and Technology Project ([2016]-19).

\section{References}

Abdel-Wahab MA, Dayarathne MC, Suetrong S, Guo SY et al. 2017 - New saprobic marine fungi and a new combination. Botanica Marina 60, 469-488.

Aiello D, Polizzi G, Crous PW, Lombard L. 2017 - Pleiocarpon gen. nov. and a new species of Ilyonectria causing basal rot of Strelitzia reginae in Italy. IMA fungus 8, 65-76.

Aptroot A, Sparrius LB, Alvarado P. 2018 - Aquacidia, a new genus to accommodate a group of skiophilous temperate Bacidia species that belong in the Pilocarpaceae (lichenized ascomycetes). Gorteria 40, 11-14.

Bakhshi M, Arzanlou M, Groenewald JZ, Quaedvlieg W et al. 2018 - Parastagonosporella fallopiae gen. et sp. nov. (Phaeosphaeriaceae) on Fallopia convolvulus from Iran. Mycological Progress https://doi.org/10.1007/s11557-018-1428-z. 
Boonmee S, Phookamsak R, Hongsanan S, Doilom M et al. 2017 - Mycosphere notes 51-101. Revision of genera in Perisporiopsidaceae and Pseudoperisporiaceae and other Ascomycota genera incertae sedis. Mycosphere 8, 1695-1801.

Braun U, Nakashima C, Crous PW, Groenewald JZ. 2018 - Phylogeny and taxonomy of the genus Tubakia s. lat. Fungal Systematics and Evolution 1, 41-99.

Chen SF, Wingfield MJ, Li GQ, Liu FF. 2016 - Corticimorbus sinomyrti gen. et sp. nov. (Cryphonectriaceae) pathogenic to native Rhodomyrtus tomentosa (Myrtaceae) in South China. Plant Pathology 65, 1254-1266.

Crous PW, Groenewald JZ. 2017 - The genera of fungi-G 4: Camarosporium and Dothiora. IMA fungus 8, 131-152.

Crous PW, Schumacher RK, Wingfield MJ, Akulov A. 2018 - New and Interesting Fungi. 1. Fungal Systematics and Evolution 1, 169-215.

Crous PW, Wingfield MJ, Burgess TI, Carnegie AJ et al. 2017 - Fungal Planet description sheets: 625-715. Persoonia 39, 270-467.

Dayarathne MC, Maharachchikumbura SSN, Jones EB, Goonasekara ID et al. 2017 Neophyllachora gen nov. (Phyllachorales), three new species of Phyllachora from Poaceae and resurrection of Polystigmataceae (Xylariales). Mycosphere 8, 1598-1625.

Dayarathne MC, Wanasinghe DN, Jones EG, Chomnunti P et al. 2018 - A novel marine genus, Halobyssothecium (Lentitheciaceae) and epitypification of Halobyssothecium obiones comb. nov. Mycological Progress https://doi.org/10.1007/s11557-018-1432-3.

Dong W, Hyde KD, Bhat DJ, Zhang H. 2018 - Introducing Aculeata aquatica gen. et. sp. nov., Minimelanolocus thailandensis sp. nov. and Thysanorea aquatica sp. nov. (Herpotrichiellaceae, Chaetothyriales) from freshwater in northern Thailand. Mycological Progress 17, 617-629.

Ertz D, Poulsen RS, Charrier M, Søchting U. 2017a - Taxonomy and phylogeny of the genus Steinera (Arctomiales, Arctomiaceae) in the subantarctic islands of Crozet and Kerguelen. Phytotaxa 324, 201-238.

Ertz D, Sanderson N, Łubek A, Kukwa M. 2018 - Two new species of Arthoniaceae from old-growth European forests, Arthonia thoriana and Inoderma sorediatum, and a new genus for Schismatomma niveum. The Lichenologist. 50, 161-172.

Ertz D, Søchting U, Gadea A, Charrier M, Poulsen RS. 2017b -Ducatina umbilicata gen. et sp. nov., a remarkable Trapeliaceae from the subantarctic islands in the Indian Ocean. The Lichenologist 49, 127-140.

Hyde KD, Chaiwan N, Norphanphoun C, Boonmee S et al. 2018 - Mycosphere Notes 169-224. Mycosphere 9, 271-430.

Hyde KD, Norphanphoun C, Abreu VP, Bazzicalupo A et al. 2017 - Fungal diversity notes 603-708: taxonomic and phylogenetic notes on genera and species. Fungal Diversity 87, 1-235.

Jaklitsch W, Baral HO, Lucking R, Lumbsch HT. 2016 - Ascomycota. In: Frey W (ed) Syllabus of plant families, 23rd edn.

Jaklitsch WM, Checa J, Blanco MN, Olariaga I et al. 2017 - A preliminary account of the Cucurbitariaceae. Studies in Mycology 90, 71-118.

Jayasiri SC, Hyde KD, Jones EG, Ariyawansae HA et al. 2017 - A new Hysteriform Dothideomycete (Gloniaceae, Pleosporomycetidae Incertae sedis), Purpurepithecium murisporum gen. et sp. nov. on pine cone scales. Cryptogamie Mycologie 38, 241-251.

Karunarathna A, Papizadeh M, Senanayake IC, Jeewon R et al. 2017 - Novel fungal species of Phaeosphaeriaceae with an asexual/sexual morph connection. Mycosphere 8, 1818-1834.

Kirk P, Cannon PF, Minter DW, Stalpers JA. 2008 - Ainsworth \& Bisby's Dictionary of the Fungi, 10th edn. CAB International, Wallingford

Li WL, Luo ZL, Liu JK, Bhat DJ et al. 2017 - Lignicolous freshwater fungi from China I: Aquadictyospora lignicola gen. et sp. nov. and new record of Pseudodictyosporium wauense from northwestern Yunnan Province. Mycosphere 8, 1587-1597. 
Liu JK, Lu YZ, Cheewangkoon R, To-Anun C. 2018 - Phylogeny and morphology of Helicotubeufia gen. nov., with three new species in Tubeufiaceae from aquatic habitats. Mycosphere 9, 495509.

Lu YZ, Boonmee S, Liu JK, Hyde KD et al. 2018 - Multi-gene phylogenetic analyses reveals Neohelicosporium gen. nov. and five new species of helicosporous hyphomycetes from aquatic habitats. Mycological Progress 17, 631-646.

Pawłowska J, Istel Ł, Gorczak M, Galera H et al. 2017 - Psychronectria hyperantarctica, gen. nov., comb. nov., epitypification and phylogenetic position of an Antarctic bryophilous ascomycete. Mycologia https://doi.org/10.1080/00275514.2017.1398575.

Senanayake IC, Crous PW, Groenewald JZ, Maharachchikumbura SSN et al. 2017 - Families of Diaporthales based on morphological and phylogenetic evidence. Studies in Mycology 86, 217-296.

Senanayake IC, Jeewon R, Chomnunti P, Wanasinghe DN et al. 2018 - Taxonomic circumscription of Diaporthales based on multigene phylogeny and morphology Fungal Diversity https://doi.org/10.1007/s13225-018-0410-z.

Senwanna C, Phookamsak R, Doilom M, Hyde KD et al. 2017 - Novel taxa of Diatrypaceae from Para rubber (Hevea brasiliensis) in northern Thailand; introducing a novel genus Allocryptovalsa. Mycosphere 8, 1835-1855.

Tanaka K, Hashimoto A, Matsumura M, Sato T. 2017 - Brevicollum, a new genus in Neohendersoniaceae, Pleosporales. Mycologia 109, 608-619.

Tanney JB, Seifert KA. 2018 - Pileospora piceae gen. et sp. nov. (Septorioideaceae, Botryosphaeriales) from Picea rubens. Mycological Progress https://doi.org/10.1007/s11557018-1423-4

Thambugala KM, Wanasinghe DN, Phillips AJ, Camporesi E et al. 2017 - Mycosphere notes 1-50: grass (Poaceae) inhabiting Dothideomycetes. Mycosphere 8, 697-796.

Tibpromma S, Hyde KD, Jeewon R, Maharachchikumbura SS et al. 2017 - Fungal diversity notes 491-602: taxonomic and phylogenetic contributions to fungal taxa. Fungal Diversity 83, 1261.

Valenzuela-Lopez N, Cano-Lira JF, Guarro J, Sutton DA et al. 2017 - Coelomycetous Dothideomycetes with emphasis on the families Cucurbitariaceae and Didymellaceae. Studies in Mycology 90, 1-69.

Wanasinghe DN, Hyde KD. 2018 - Nomenclatural novelties: Index Fungorum no. 357.

Wanasinghe DN, Hyde KD, Jeewon R, Crous PW et al. 2017 - Phylogenetic revision of Camarosporium (Pleosporineae, Dothideomycetes) and allied genera. Studies in Mycology 87, 207-256.

Wanasinghe DN, Phukhamsakda C, Hyde KD, Jeewon R et al. 2018 - Fungal diversity notes 709839: taxonomic and phylogenetic contributions to fungal taxa with an emphasis on fungi on Rosaceae. Fungal Diversity 89, 1-236.

Wijayawardene NN, Hyde KD, Divakar PK, Rajeshkumar KC. 2018 - Notes for genera updateAscomycota: 6616-6821. Mycosphere 9, 115-140.

Wijayawardene NN, Hyde KD, Rajeshkumar KC, Hawksworth DL et al. 2017 - Notes for genera Ascomycota. Fungal Diversity 86, 1-594.

Wijayawardene NN, Hyde KD, Wanasinghe DN, Papizadeh M et al. 2016 - Taxonomy and phylogeny of dematiaceous coelomycetes. Fungal Diversity 77, 1-316.

Yang J, Maharachchikumbura SS, Liu JK, Hyde KD et al. 2017 - Pseudostanjehughesia aquitropica gen. et sp. nov. and Sporidesmium sensu lato species from freshwater habitats. Mycological Progress https://doi.org/10.1007/s11557-017-1339-4.

Zeng XY, Hongsanan S, Hyde KD, Putarak C, Wen TC. 2018 - Translucidithyrium thailandicum gen. et sp. nov.: a new genus in Phaeothecoidiellaceae. Mycological Progress https://doi.org/10.1007/s11557-018-1419-0.

Zhurbenko MP, Frisch A, Ohmura Y, Thor G. 2015 - Lichenicolous fungi from Japan and Korea: new species, new records and a first synopsis for Japan. Herzogia 28, 762-789. 
Zhurbenko MP, Pino-Bodas RA. 2017 - A revision of lichenicolous fungi growing on Cladonia, mainly from the Northern Hemisphere, with a worldwide key to the known species. Opuscula Philolichenum 16, 188-266. 